\title{
EL LEXEMA VERBAL TRANSITIVO: CRITERIOS DE SELECCIÓN DE UN CORPUS NUCLEAR PARA LA ENSEÑANZA/APRENDIZAJE DE UNA L2
}

\author{
Luis Rodríguez García \\ Departamento de Filología Francesa e Inglesa \\ Universidad de Córdoba
}

\begin{abstract}
RESUMEN: este artículo supone una aplicación didáctica de los postulados de los enfoques funcional y cognoscitivo de la lengua, que conceden al componente léxico un papel fundamental en el análisis lingüístico. Propone criterios para la elaboración de un syllabus léxico funcional nuclear para la enseñanza del inglés como L2, que vendría representado por un corpus de verbos transitivos prototípicos. Se aducen razones lingüísticas y cognoscitivas que justifican esta opción metodológica evidenciando, en primer lugar, que en tales verbos se da la confluencia de prototipicalidad de los diversos niveles de lengua (léxico-gramático-discursivo); y, en segundo lugar, que los modelos cognoscitivos idealizados que los representan, no sólo tienen un carácter universal, sino que estructurasn y determinan el funcionamiento del léxico mental. Tales propiedades del corpus propuesto, presentadas en mapas cognoscitivos semánticos, propiciarían el acercamiento entre L1 y L2 y podrían, por tanto, acortar la ruta cognoscitiva en el proceso de adquisición del inglés como L2, mediante la transferencia de los recursos cognoscitivos/lingüísticos que ya se poseen en el español como L1.
\end{abstract}

ABSTRACT: From the functional and cognitive approaches to language, the lexical component represents the main element in the linguistic analysis. The present article supposses the application of this principle to the teaching of English as L2 through the establishment of a series of criteria for the selection of a nuclear functional lexical syllabus. These criteria coincide with a corpus of prototypical transitive verbs, as it is revealed by linguistic and cognitive reasons: first, in such verbs there are a confluence of prototipicallity referred to the three levels of language as communication (lexical-grammatical and discoursive); second, the idealized cognitive models (ICMs) these verbs are represented by are not only universal buy, furthermore, they structure and determine the mental lexicon operation. These properties presented in a cognitive-semantic-map frame could shorten the cognitive distance between Spanish and English by transfering the cognitivelinguistic resources which learners already possess in their first language.

\section{Objetivos}

Los enfoques funcional-pragmático y cognitivo de la lengua de las últimas décadas han traído consigo una reivindicación del componente léxico como elemento esen- 
cial del análisis lingüístico, con amplias posibilidades pedagógicas. Este giro copernicano dado en los modelos de lengua ha hecho que en el ámbito de la lingǘstica aplicada a la enseñanza de una L2 se considere el vocabulario como el elemento esencial que dirige todo el proceso de su adquisición: la palabra es la unidad central de la descripción de la lengua y el lexicón, por tanto, es el dominio informativo por excelencia.

Este avance teórico formulado por las distintas disciplinas lingüísticas y metodologías de enseñanza con respecto al conocimiento léxico, requiere una descripción integradora del mismo y unas aplicaciones que respondan a la naturaleza de la propia lengua y a las necesidades reales de sus usuarios, también en lo que concierne al aprendizaje de una L2. Para nosotros, el Modelo Lexemático Funcional (MLF) supone una respuesta adecuada a estas demandas ${ }^{1}$ La pregunta es icómo puede organizarse un syllabus léxico nuclear que respondiendo a estas características globarizadoras del lexicón suponga un instrumento adecuado de adquisición de una L2?; es decir, que, aunque partiendo del nivel léxico, considere el discurso como la unidad comunicativa por excelencia de la lengua, integrando en su seno los diversos niveles de lengua como exponentes de las tres metafunciones lingüísticas señaladas por Halliday (ideacional, interpersonal y textual) ${ }^{2}$

El presente artículo supone una alternativa a los tradicionales criterios de frecuencia y limitación del rol del vocabulario en la enseñanza de una L2, proponiendo una fundamentación para la elaboración de un corpus funcional de lexemas verbales prototípicamente transitivos destinado a la enseñanza/aprendizaje del inglés como L2. Se sugieren razones lingüísticas y cognitivas que justifican esta opción metodológica, evidenciando, en primer lugar, que la confluencia de prototipicidad de los diversos nieveles (lexico-gramático-discursivo) coincide con el criterio de selección señalado; y, en segundo lugar, que, de acuerdo con la Teoría Prototípica defendida por la semántica cognitiva, los modelos cognitivos idealizados (MCIs) que representan a ambos fenómenos, además de tener un carácter universal que propicia el acercamiento entre una L1 y una L2, estructuran y determinan el funcionamiento del léxico mental y, pueden, por tanto, acortar la ruta cognitiva en el proceso

1. El Modelo lexicológico Lexemático-Funcional (MLF) propuesto por el Profesor Martín Mingorance $(1984,1987,1990,1994)$ se presenta como un modelo ecléctico que fundamenta su análisis lingüístico en la consideración fundamental de que la lengua está organizada jerárquicamente en dos ejes básicos: el eje paradigmático, que establece relaciones de selección entre los lexemas, y el eje sintagmático, que determina las relaciones de solidaridad o complementación; ambos ejes se complementan y su intersección establece una jerarquía de esquemas cognitivos que posibilitan la organización y el funcionamiento intelectivo que hace viable nuestra relación con el entorno.

2. Este principio jerárquico ha quedado bien establecido por la GF de Dik: The study of syntax and semantics should be carried out against the background of the pragmatic conventions which determine the use of language in verbal interaction. Syntax is subservient to semantics, and semantics is subservient to pragmatics, (Dik, 1980:46). 
de adquisición de una L2, mediante la transferencia de los recursos cognitivos/lingüísticos que ya se poseen en una L1.3

\section{El lexema verbal entre el léxico y la gramática}

\section{(i) Importancia comunicativa del verbo}

El análisis de la estructura y funcionamiento del léxico mental desde la perspectiva del MLF confirma que el lexema verbal puede considerarse con toda razón el elemento esencial y determinante de la estructura oracional. Ésta es, a su vez, la depositaria natural de la proposición (nivel léxico-gramatical), que se considera como la unidad básica informativa de la lengua humana, teniendo como finalidad típica transmitir una información específica (contenido), que un hablante (emisor) quiere comunicar a un oyente (receptor), en un determinado contexto discursivo (nivel discursivo/pragmático).

Centraremos nuestro análisis en los paradigmas funcional-cognitivo. Así, desde el modelo funcional sistémico de Halliday, el escenario semántico prototípico para la representación conceptual de la proposición es constante y relativamente simple: la mayoría de las lenguas codifica las experiencias con una fórmula semántica que contiene, potencialmente, cuatro elementos o componentes funcionales: Participante (entidad involucrada de alguna manera en el proceso), Proceso descrito por un verbo (material, mental, relacional y existencial, principalmente), Atributo (cualidad o característica de los participantes, y Circunstancia (cualquier contingencia que afecte al proceso en su totalidad). De todos estos componentes, es el verbo el elemento esencial que determina la propia naturaleza del proceso.

Dik (1978b, 1989), por su parte, explica cómo se estructura jeráquicamente el enunciado de estas proposiciones a través de la construcción de lo que él denomina "predicaciones subyacentes", que , en realidad, son también estructuras semánticas que más tarde se convierten en las expresiones lingüísticas mediante la aplicación de "reglas de expresión". En efecto, Dik parte del concepto básico del "marco predicativo" (predicative frame), que tiene al verbo como el elemento central que determina la

3. Estamos sugeriendo estrategias procedimentales en la línea de Carter (1986) "core vocabulary", Widdowson (1983) "procedural vocabulary" o Robinson (1988), quien afirma que tales corpus can be seen as the lexical equivalent of Halliday's transitivity system, which at the clause level, serves to represent 'the categories of our interpretation of experience'. (Robinson, 1988:231). En efecto, consideramos que establecer listados de contenidos, dada la inmensidad de la tarea, es siempre, en el mejor de los casos, como "a drop in a bucket"; por ello, es necesario recurrir a este tipo de estrategias (en concreto, este corpus tendría al menos un alto poder exponencial), y, en correspondencia con la concepción funcional de la lengua, la categorización propuesta incluye además el nivel discursivo. 


\section{LUIS RODRÍGUEZ GARCÍA}

naturaleza de los "estados de cosas" (states of affairs) y, en torno al cual, se construye la predicación mediante la adición de diversos operadores y satélites. ${ }^{4}$

Desde la perspectiva de la gramática funcional tipólogica, Givón (1984) expresa esta misma idea acerca de la importancia decisiva del lexema verbal, afirmando que en términos funcionales puede establecerse una tipología universal de procesos expresados por oraciones simples en relación, justamente, con la naturaleza de los verbos y el marco predicativo que ellos codifican. Los componentes formales de estas predicaciones serían: (i) the propositional frame ("semantic grid"); and (ii) the actual lexical items filling those type of slot (Givón, 1984:86). La combinación de ambos componentes genera el significado proposicional de una oración y comporta, a su vez, consecuencias sintácticas.

Pese a las diferencias terminológicas empleadas por los diversos enfoques funcionales, el fenómeno experiencial es percibido en todos como una totalidad y hay coincidencia en que de las diferentes categorías léxicas que constituyen los elementos básicos que configuran las funciones semánticas y los roles sintácticos en una proposición, es el verbo el que asume con diferencia la función determinante en el tipo de proceso y su comportamiento en todos los niveles de lengua. ${ }^{5}$

Este hecho, con diferente énfasis, es también asumido por otros paradigmas lingüísticos. Así, por ejemplo, Allerton (1982) reconoce el rol sustancial del verbo en la constitución de una tipología de oraciones. ${ }^{6}$ Aitchison (1987:111), por su parte, afir-

4. Dik considera que la predicación resultante se expresa mediante el marco predicativo que, en el caso del predicado verbal, puede considerarse como un esquema cognitivo con casillas argumentales disponibles para representar infinidad de estados de cosas. Así pues, las predicaciones tienen una estructura lingüística propia con información sintáctica, semántica y pragmática, así como restricciones de uso, independiente del contenido léxico concreto, y determinada, en parte, por la estructura semántica que el lexicón fija para los lexemas verbales, por donde podría decirse que constituyen una tipología de carácter universal.

Dik da un ejemplo concreto, supongamos que queremos generar la frase: The old man gave the book to John in the library. Para llegar al tipo de predicación que subyace en esta frase, necesitamos el marcopredicativo básico del verbo give que está codificado en el lexicón de la siguiente manera:

givev(x1:animate(x1))Ag(x2)Go(x3:animate(x3))Rec

Así, give está codificado como un predicado verbal (V), que toma tres argumentos indicados por las variables x1, x2, x3, con las funciones semánticas de Agente (Ag), Meta (Go), y Recipiente (Rec), estando el Agente y el Recipiente restringidos (=selection restriction) a términos animados. Este marco predicativo básico tendría que ser ampliado con un argumento opcional de circunstancia de lugar, Locación (Loc); para rellenar este marco necesitaríamos cuatro términos apropiados que reuniesen los requisitos de inserción requeridos. Este proceso daría lugar a un output o predicación, que, tras pasar por las reglas de expresión, daría lugar a una expresión lingüística correcta. Cada marco predicativo del lexicón está asociado con un número de postulados de significado (=meaning postulates), a través de las cuales se relaciona semánticamente con los otros predicados de la lengua.

5. Heringer (1989) define al verbo como el centro rector o núcleo estructurador de la oración, correspondiéndole una función organizativa. La estructura básica del verbo predetermina la actantificación. "Un verbo es como encender una luz en un cuarto oscuro. De golpe, nace todo un escenario".

6. Verb is needed as an essential nucleus for the major type of sentence patterns in the language (...). One aspect of this centrality of the verb in the sentence is that the kind of verb that is selected for a particular sentence determines the basic structure of that sentence (Allerton, 1982:5). 
ma que the verb is the pump of the sentence; Miller \& Fellbaum (1991:214) expresan el mismo criterio cuando reconocen que the verbs are arguibly the most important lexical category of a language; y, por no hacer muy largo el rosario de citas que podrían corroborar la importancia decisiva del lexema verbal en la estructura del léxico mental y su funcionamiento reflejado en las expresiones lingüísticas, terminemos con la afirmación de Gleitman (1990), a verb forces to take the number of arguments, the type of arguments, a focus on the cause or effect, and the choice of agent and affected entity when more than one is cognitively possible.

Siguiendo este criterio, son muchos los lingüistas que han presentado modelos proposicionales de acuerdo con el tipo de verbos que pueden aparecer en posición central organizando el resto de los elementos (Chafe, 1970; Fillmore, 1968). En efecto, los verbos dominan la oración y dictan su estructura. Son ellos los que especifican la naturaleza sintáctica de los procesos experienciales y suelen aportar la mayor carga semántica en el proceso informativo presentado por la predicación; es decir, fijan no sólo el tipo y número de entidades participantes a que ha lugar, sino sus roles sintácticos y semánticos: this syntactic and semantic information is generally thought to be part of the verb's lexical entry, that is to say, part of the information about the verb that is stored in speaker's mental lexicon (Fellbaum, 1990:278).

Pero la importancia del lexema verbal no puede restringirse tan sólo a los niveles significativos conceptual y proposicional sino que, de acuerdo con la teoría de los esquemas y la consideración comunicativa del lenguaje, esta importancia debe también proyectarse hacia el nivel discursivo, es decir, al significado pragmático del texto. En efecto, es nuestra intención sugerir que el corpus diseñado incluye este macronivel como justificación de su potencial comunicativo. ${ }^{7}$

\section{(ii) Naturaleza semántica del verbo}

Habiendo puesto de manifiesto que la importancia del lexema verbal descansa por igual en los diversos niveles lingüísticos, interesa, no obstante, analizar un aspecto puesto de relieve de manera especial por la GF y la gramática cognitiva (GC): su naturaleza semántica. En efecto, los rasgos semánticos confieren una cierta gradación de pertenencia más o menos prototípica a esta categoría léxica que puede servir como criterio de selección de un corpus nuclear. ${ }^{8}$

7. Esta es también la línea de investigación sugerida por McCarthy y Carter (1994) to examine the significance of as many choices as possible as the lexico-grammatical level which seem to have implications for the creation of the macro-levels features (...) the foundation of a discourse-based view of language and of language teaching, (McCarthy y Carter, 1994:90).

8. Para expresar esta realidad de la lengua se ha recurrido a la metáfora de considerar el idioma como una especie de iceberg del cual sólo se ve la parte superior, es decir, la menos considerable, mientras que la parte mayor se encuentra sumergida bajo el agua; esta última corresponde a la parte semántica. 
No es fácil responder a la pregunta ¿qué es un posible verbo? Desde la perspectiva de estos paradigmas se ha insistido en la conveniencia de proceder a un estudio semántico que revele la naturaleza significativa del verbo y cómo se pueden aislar estos lexemas. En este sentido, Croft (1990) afirma que la respuesta a la pregunta anterior requiere una mayor atención que definir la naturaleza del sustantivo, en cuanto que la mayoría de los "objetos" que constituyen esta categoría gramatical están ya individualizados, ocupando un espacio propio, físico o mental. Así, su existencia e individualización, al menos en los casos prototípicos realizados por objetos concretos, queda evidenciada por la simple percepción. Los verbos, en cambio, al representar procesos o estados de cosas, ofrecen una mayor dificultad para ser categorizados. Estos eventos no están individualizados claramente en las coordenadas espacio-temporales.

Según Langacker (1987:244), a la pregunta sobre la naturaleza semántica del verbo se puede contestar diciendo que, como cualquier otra categoría gramatical, un verbo es una expresión simbólica cuya referencia semántica en lugar de constituirla un "objeto", como en el caso del sustantivo, la constituye un proceso.

Desde la Gramática Funcional Tipológica, Givón (1984) ha propuesto un parámetro de gran coherencia para establecer una clasificación general de las palabras en las categorías gramaticales: the time-stability-scale. Siguiendo este criterio, experiencias que permanecen relativamente estables a lo largo del tiempo tienden a ser lexicalizadas por la lengua humana como sustantivos. Los sustantivos más prototípicos son aquellos que denominan entidades concretas, físicas y compactas, hechas de una sustancia durable y sólida. Por supuesto, su estabilidad temporal es obviamente un asunto de grados, puesto que los seres animados cambian más rápidamente que los inanimados. En el otro extremo de la escala fenomenológica-léxica, nos encontramos con conjuntos experienciales (experiential clusters) que denotan "cambios rápidos" en el estado del universo. Estas experiencias son prototípicamente eventos y acciones y la lengua tiende a lexicalizarlos como verbos. Los miembros de esta categoría son muchos más abstractos que los sustantivos, y, por supuesto, también admiten gradación en su prototipicidad. Así, de acuerdo con el principio defendido por la escala-deestabilidad-temporal, los verbos tienden a codificar experiencias menos estables, principalmente acciones rápidas, eventos transitorios, y transformaciones de estados. La transitoriedad, pues, constituye el criterio de prototipicidad por el que la categoría se caracteriza, cubriendo un espacio semántico en la escala de la estabilidad temporal distinto a las otras categorías lexemáticas. Dependiendo del grado o nivel ocupado por el verbo en esta escala, Givón (1984) distingue tres tipos de verbos de mayor a menor prototipicidad: (i) los que representan acciones instantáneas (hit, shoot, kick); (ii) los que representan una actividad o proceso más lento, (por ejemplo: "read"); y (iii) los que representan un estado (no-cambio) o un proceso muy lento de transformación, (por ejemplo: know, understand, like, etc.). 
Por incluir otras perspectivas funcionales, diremos que Halliday (1985) establece unos parámetros de clasificación muy similares a los analizados y distingue también tres tipos fundamentales de procesos que responden a la naturaleza de los verbos que los constituyen: material (acción), mental (conocimiento, percepción y afecto), y relacional (estados o transformaciones). Dik, que distingue sólo tres categorías léxicas (sustantivo, adjetivo y verbo), clasifica esta última de acuerdo con los rasgos de dinamicidad y control, a los que en el modelo del 89 añade los de telicidad, instanteneidad y experiencia. La combinación de estos rasgos da lugar a la siguiente tipología de Estados de Cosas: [Situación (-D): Posición (-D, +C), Estado (-D, -C)], [Evento $(+\mathrm{D})$ : Acción $(+\mathrm{D},+\mathrm{C})$, Realización $(+\mathrm{D},+\mathrm{C},+\mathrm{T})$, Actividad $(+\mathrm{D},+\mathrm{C},-\mathrm{T})]$, [Proceso $(+\mathrm{D},-\mathrm{C})$ : Cambio $(+\mathrm{D},-\mathrm{C},+\mathrm{T})$, Dinamismo $(+\mathrm{D},-\mathrm{C},-\mathrm{T})]^{9}$

En definitiva, resumiendo las posiciones hasta ahora analizadas, una definición de la categoría verbal en términos cognitivo/funcionales, bien sea a través de la escala de estabilidad temporal (time stability scale), que propone Givón (1984), bien sea a través del perfil temporal (the temporal profile), propuesta por Langacker (1987), bien sea en la manera como se conjugan los rasgos de dinamicidad y control (Dik (1978), lo importante es que en todos los casos, la categorización propuesta coincide en lo fundamental con nuestra experiencia psicológica básica de que la significación semántica fundamental de un verbo viene caracterizada por la forma dinámica como se conceptualiza un proceso, su significado último es designar una relación de cambio de unos elementos dentro de las coordenadas espacio/temporales. Tal constatación implica la idea de que la categoría verbal simboliza paradigmáticamente "perceptos" que carecen de "estabilidad temporal" y, consiguientemente, denotan acciones o eventos concretos, kinéticos, visibles y efectivos. De acuerdo con el grado que estos rasgos semánticos y morfosintácticos prototípicos sean reflejados por lexemas verbales concretos, puede establecerse una escala de prototipicolidad para la categoría. ${ }^{10}$

9. Desde la perspectiva de la gramática cognitiva se observa, igualmente, una gran similitud con el análisis del paradigma funcional analizado. Así, Langacker (1987), siendo igualmente consciente de la dificultad de una demostración empírica, coincide con Givón en que psicológicamente, existe una conexión innegable entre la categoría verbal y la noción de temporalidad; él define la naturaleza del proceso que constituye cualquier verbo diciendo que, the processual predication has a positive temporal profile; i.e. its evolution through conceived time is scanned in sequential fashion (Langacker, 1987:244). Añade que, para que un verbo constituya un proceso, además de esta "positiva orientación temporal", su conceptualización lingüística demanda otras exigencias adicionales que podrían resumirse de la siguiente manera: (i) categorization as a process requires that the profile not be restricted to a single state; (ii) a process is relational, (i.e.) it profiles interconections; (iii) it requires a sequential scanning processing model. (Cf. Langacker, 1987:245ss).

10. Para Hopper y Thompson (1980:155), la prototipicidad de un verbo depende no sólo de la asignación de propiedades semánticas verificables independientemente, sino también, y quizás más crucialmente, de su función lingüística en el discurso. 


\section{La Transitividad entre el proceso de "gramaticalización" y la construcción del discurso}

De la misma manera que en el nivel léxico se considera al verbo como la categoría más importante, el fenómeno lingüístico de la Transitividad es visto desde el paradigma cognitivista como la estructura gramatical prototípica germen del significado proposicional. Siendo así, que la prototipicidad verbal viene a coincidir con los rasgos lingüísticos que caracterizan la Transitividad. ${ }^{11}$

Aunque con diferente énfasis, el estudio de la Transitividad ha tendido a ser considerado por los diferentes paradigmas desde tres niveles o perspectivas lingüísticas distintas: lexemática, paradigmática y sintagmática, (Cf. Cano Aguilar, 1981). Conviene, no obstante, abordar la Transitividad desde el nivel pragmático o discursivo como un fenómeno globalizado. Este nivel, que sistemáticamente ha sido soslayado, ha sido puesto de manifiesto por la GF y la GC, lo que a nuestro juicio constituye el marco integrador que justifica la práctica universalidad del fenómeno. En efecto, si desde el punto de vista funcional la lengua se caracteriza por su potencial comunicativo, ¿puede un fenómeno lingüístico tan crucial para la estructura de la gramática de la mayoría de las lenguas, justificarse autónomamente (ya sea en el nivel sintáctico o semántico, o en ambos a la vez) o tendrá necesariamente que referirse a una función comunicativa de la misma entidad, es decir, de carácter universal y de importancia decisiva en el proceso discursivo?

Entendiendo que la GF representa un modelo de lengua orientado cognitivamente y dada la importancia que este paradigma ha tenido para la historia de la linguística de los últimos veinte años, conviene que, siquiera sea someramente, abordemos el tratamiento que la GF ha dispensado al fenómeno de la Transitividad.

11. Desde la perspectiva semántica, Croft (1990) propone “The Causal Hypothesis”, ampliamente aceptada hoy en día, para defender el modelo cognitivo utilizado en la categorización de los procesos verbales. A esta hipótesis se le ha denominado también "The Causal Chain”, y viene a defender que los procesos denotados por la categoría verbal reflejan básicamente the transmission of force through individuals to other individuals, whether through contact or "action at the distance" (Croft, 1990:50).

En efecto, el mundo parece funcionar mediante una red de causalidades extremadamente compleja de la que sólo percibimos fragmentos. Los verbos, que constituyen sus significados mediante la combinación de los componentes semánticos y las estructuras sintácticas que ellos demandan, adquieren sentido en el esquema cognitivo que los identifica como un "eslabón" de esa cadena causal que constituye el "continuum" de nuestra experiencia del mundo. Así, al rasgo prototípico de dinamismo o cambio que antes señalábamos para su comprensión semántica, habría que añadir el principio de fuerza-causando-un-cambio para su categorización como lexema verbal prototípico. Como veremos a continuación, éste es en esencia el esquema prototípico de la construcción gramatical caracterizada como Transitividad. En efecto, aun admitiendo que se dan realizaciones procesuales más periféricas, el proceso no-marcado o prototípico lo constituye la transitividad o causalidad, the structure of events in the world was a vast causal network that human beings must break down in some way for apprehension, (...) and events requiring external agents are going to be unmarked causatives, (Croft, 1990:49). 
Halliday (1985) aborda el fenómeno de la Transitividad al hablar de la función ideativa o representacional como una propiedad que la lengua nos brinda para conceptualizar y describir las experiencias: lo que ocurre en nuestro entorno y en nuestro interior y constituye el contenido del significado lingüístico expresado en las oraciones (transitive structures). Así pues, una primera definición consideraría la oración transitiva como la representación lingüística (nivel sintáctico) de los procesos (nivel semántico) que reflejan la construcción mental de la realidad externa e interna, dándoles sentido (nivel pragmático).

¿Qué sentido tiene afirmar que una oración representa un proceso? Nuestra concepción de la realidad nos dice que ésta forma un "contínuum" (consists of "goingson”). Estos sucesos continuados son distribuidos en el sistema semántico de la lengua y se expresan a través de la gramática de la oración. El aspecto experiencial del significado constituye el sistema de Transitividad, Transitivity specifies the different types of process that are recognized in the language, and the structures by which they are expressed, (Halliday, 1985:101).

De los diversos tipos de procesos (materiales, mentales, relacionales, etc.), el proceso material es el que mejor representa la estructura prototípica del sistema de la Transitividad:

Material processes are processes of "doing”. They express the notion that some entity "does" something-which may be done "to" some other entity.(...) Alternatively, the other entity may be one that brought into being by the process. (...) We can thus distinguish between a "doing to", or DISPOSITIVE type and a "bringing about”, or CREATIVE type of material process (Halliday, 1985:104).

Así pues, la estructura transitiva de este proceso requiere la presencia de un Agente (=Actor or logical subject) que representa a la entidad o primer Participante que realiza la acción y un segundo Participante, Meta (=Affectum (Goal) or logical object) que representa a la entidad afectada por la acción.

Si para Halliday la Transitividad se realiza de modo prototípico en el "proceso material", en la GF de Dik (1978, 1989) viene a coincidir, fundamentalmente, con el estado de cosas que indica actividad (+ dinamismo, + control). Goossens (1990), en un intento de integrar la noción de Transitividad como se concibe por la GC en las estructuras subyacentes de la GF, afirma que Goal is the key to prototypical transitivity in FG (Goossens, 1990:62). Entendiendo Meta (Goal) como una función semántica en los términos definidos por Dik: Goal is the entity affected or effected by the operation of some controller (Agent/Positioner) or Force (Dik, 1989:103). Así, un estado de cosas que contiene un rol semántico "Meta" puede ser visto como la más cercana realización a la Transitividad prototípica: In line with the cognitive definition of transitivity, construing an argument or satellite as Affected, implies that the state of affairs is (often metaphorically) conceived as involving an energized transfer of energy from the first argument to some other asymmetrical entity, (Goossens, 1990:66). 
Desde la perspectiva de la semántica cognitiva, (Lakoff, 1987; Langacker, 1987, 1990; Johnson, 1987; Taylor, 1989) la Transitividad constituye una noción crucial para entender un amplio abanico de interrelaciones que se dan en casi todas las lenguas. El definir la Transitividad como la construcción gramatical prototípica trae como corolario inmediato el admitir que debe generar los importantes efectos que la Teoría Prototípica postula para cualquier prototipo: estructurar básicamente la categoría a la que pertenece (en este caso, el esquema cognitivo conceptual de la Transitividad se considera como esquema básico de la construcción gramatical) y, en segundo lugar, dar sentido y contribuir al desarrollo de la categoría (en este caso, facilitar por extensión metafórica o metonímica la incorporación de nuevos miembros de estructuras gramaticales de carácter simbólico). Su importancia conceptual y lingüística es, pues, evidente y de grandes consecuencias didácticas como expondremos.

Siguiendo la definición de construcción lingüística dada por Taylor, (1989:198), Cognitive Grammar (...) views a construction as the pairing of a specification of form with a specification of meaning, abordaremos brevemente la forma de la construcción transitiva, para analizar a continuación los criterios semánticos que definen el fenómeno:

The syntactic properties of the "transitive" construction may be presented by the formula $N P_{1} V_{\text {TRANS }} N P_{2}$ where $N P_{1}$ and $N P_{2}$ stand for the subject and direct object, and $V_{\text {TRANS }}$ is a transitive verb. In its prototypical instantiations, both NPs have specific reference, while the verb is "realis", (Taylor, 1989:207).

Desde el punto de vista semántico, el esquema prototípico de la Transitividad vendría conformado por una relación de transferencia entre dos participantes. El tipo de relación y las características de los argumentos que integran esta totalidad tiene una serie de rasgos prototípicos que podría analizarse en los siguientes términos:

(i) El verbo: indica la modalidad de la relación. Debe consistir en una acción -los estados no pueden transferirse- (KINESIS); referirse a un hecho puntual -la inmediatez evidencia mejor el efecto que el tránsito temporal que va de la incepción a la complexión de la acción-(INSTANTENEIDAD); supone un logro o consecución -la transferencia es más evidente en el aspecto perfectivo- (TELICIDAD); la relación entre los argumentos debe ser de contacto físico directo y presentar unos efectos reales-lo imaginario o hipotético es aún contingente-(MODALIDAD).

(ii) El primer participante (SUJETO/AGENTE): es típicamente el iniciador y responsable de la relación establecida. Actúa de forma consciente y voluntaria y controla la acción.

(iii) El segundo participante (OBJETO/PACIENTE): es típicamente algo inanimado; es el referente del agente que recibe pacientemente el efecto de la acción promovida por el primer argumento. El rasgo más típico del efecto de esta acción es su perceptibilidad, el hecho de que pueda ser observada con facilidad por cualquier observador.

Analizados los rasgos prototípicos, conviene destacar dos propiedades de la Transitividad, aparentemente encontradas pero complementarias: su globalidad y su 
carácter gradual. En efecto, respecto a la primera, aunque metodológicamente pueda analizarse en sus partes, la Transitividad, como cualquier otro esquema cognitivo, es vista como una propiedad global que afecta a los tres niveles de lengua que se manifiestan en cualquier predicación. No obstante, la variación de cualquiera de los rasgos prototípicos marcados para los diversos componentes que integran la relación, origina automáticamente un efecto de gradación. Ambas propiedades han sido analizadas en forma de hipótesis por Hopper y Thompson, (1980):

(i) La primera hipótesis (Transitivity Hypothesis) defiende la correlación entre las propiedades semánticas que determinan la Transitividad y las propiedades sintácticas en las que se manifiestan, o dicho de otra forma, correlations between morphosyntactic signals and semantic interpretations, (Hopper y Thompson, 1980:255). ${ }^{12}$

(ii) La segunda hipótesis defiende la importancia pragmática de la Transitividad evidenciando una correlación entre centralidad del mensaje (grounding) y Transitivi$\mathrm{dad}$, without the connection to a communicative function, the separate components of the Transitivity relationship have only an arbitrary relationship to each other; we lack a reason why these semantic-grammatical components, rather than others, should be selected, (Hopper y Thompson, 1980:280).

Para Hopper y Thompson, el fenómeno lingüístico de carácter pragmático conocido como (grounding) es el marco funcional universal que subyace en el fenómeno, también universal, de la Transitividad. ${ }^{13}$ En efecto, en el proceso de comunicación el hablante debe seleccionar una información y estructuras pertinentes a sus propósitos comunicativos y a las necesidades perceptivas del oyente. No existe una relación lógica directa entre los hechos y su conceptualización, (Cf. más adelante ejemplos dados por Taylor y Langacker); para que se dé la comunicación se requiere un proceso muy selectivo en el que algunas partes reciben mayor relevancia a propósito del discurso (foreground) y otras, en cambio, contribuyen a la finalidad comunicativa de forma subsidiaria aportando detalles, comentarios, etc. (background):

The foregrounded (clauses) comprise the backbone or skeleton of the text, forming its basic structure; the backgrounded clauses put flesh on the skeleton, but are extraneous to its structural coherence, (Hopper y Thompson, 1980:281).

Así como existen rasgos morfosintánticos en determinadas lenguas que contribuyen al efecto comunicativo background, la centralidad del mensaje (foreground) en lenguas como el inglés no es transmitida por un simple rasgo morfosintáctico, but from a cluster of properties (...) precisely that which characterizes high Transitivity (Hopper y Thompson, 1980:282).

12. If two clauses ( $a$ ) and (b) in a language differ in that $(a)$ is higher in Transitivity according to any of the features, then, if a concomitant grammatical or semantic difference appears elsewhere in the clause, that difference will also show (a) to be higher in Transitivity, (Hopper y Thompson, 1980:255).

13. Este fenómeno (foregrounding/backgrounding) se considera como un universal lingüístico que se origina como consecuencia de dar mayor o menor relieve a ciertas partes de la comunicación, por lo que quizás, además de a razones pragmáticas también responda a funciones psicológicas. 
En resumen, igual que desde el punto de vista funcional la lengua se justifica por su potencial comunicativo, la función pragmática de la Transitividad como construcción esencial en el sistema de lengua consiste en enfatizar y revelar el contenido básico/nuclear del mensaje, de ahí que las posibilidades de que una oración alcance una posición central en el discurso estén en relación con la posición que el lexema verbal ocupe en la escala de la Transitividad, con lo que este fenómeno alcanza su clave explicativa no tanto en los hechos sintácticos o semánticos como en los pragmáticos o comunicativos. ${ }^{14}$

\section{Potencial didáctico del lexema verbal prototípicamente transitivo para la enseñanza/aprendizaje de una L2}

La importancia semántica, sintáctica y pragmática de los verbos transitivos prototípicos conlleva un indudable potencial didáctico. Ya se sabe, que sugerir aplicaciones pedagógicas desde teorías es siempre algo peligroso y que debe hacerse con ciertas precauciones. Aunque hemos tratado de demostrar que nuestras sugerencias están hechas desde una sólida fundamentación psicolingüística y responden a una seria investigación empírica en curso, la validez de la propuesta tiene aún que ser validada por aplicaciones en distintos contextos pedagógicos. Por ello, una de estas precauciones nos lleva a admitir de entrada que todas las generalizaciones didácticas son decisiones metodológicas que, aunque en absoluto ignoran el gradualismo del sistema de la lengua que sólo se resuelve y concreta en el nivel de interpretación de cada contexto, somos conscientes que al establecer criterios tajantes para la selección del corpus se peca inevitablemente de generalización y abstracción para hacer controlable la multitud de hechos diferenciales, pero ello no obsta a que tales decisiones puedan ser implementadas con otros criterios pragmáticos que incluyan "usos figurados" y extensiones metafóricas que revelen la macrored que constituye el lexicón. Dicho esto, lo hasta ahora expuesto trae como corolario:

i) respecto de la importancia del verbo en el proceso de adquisición de una lengua, ya fue puesta de relieve por la línea de investigación abierta por Bloom (1970) sobre el habla espontánea de los niños y cómo éstos interpretan significativamente el mundo y lo proyectan en categorías lingüísticas que sólo pueden establecerse por referencia a la unidad oracional que determina el verbo. Aunque posteriormente los traba-

14. A este particular, resulta muy sugerente la tesis de la "encapsulación" de Sinclair para justificar cómo la coherencia textual puede depender del hilo conductor trazado por la Transitividad, there is support in the details of text organization for the view that each new sentence takes over the status of "state of the text", and therefore that the previous sentence relinquishes that role.. (...) each new sentence encapsulates the previous one by an act of reference. By referring to the whole of the previous sentence, a new sentence uses it as part of the subject matter. (...) The regularity of this mechanism lends support to the view that each successive sentence has a kind of communicative autonomy, (Sinclair, 1994:7). 
jos de Bowerman (1973) y Slobin y Bever (1982) revelaron datos interlingüísticos que avalaron esta tesis y sirvieron para generalizarla, sorprendentemente, no puede decirse que, en esta ocasión, los esfuerzos de los lingüistas hayan sido secundados por aplicaciones didácticas de estos descubrimientos a la enseñanza de una L2.15 Por ello, constituye sin duda un campo de sumo interés científico el estudio del verbo desde esta perspectiva.

(ii) en cuanto a la importancia de la Transitividad en la adquisición de una lengua se refiere, fue Slobin (1980) quien puso de manifiesto que en las primeras experiencias lingüísticas del niño, cierta constelación de circunstancias en el mundo llegan a adquirir el estatus de prototypical transitive events y dan lugar a la construcción de frases típicamente transitivas (sujeto-verbo-objeto): un agente, preferente humano, lleva a cabo una acción que afecta a un paciente. Taylor lo explica mediante el siguiente ejemplo: el hecho de que la acción de un niño golpeando una pelota sea percibida como un hecho puntual, unitario, en lugar de descomponerlo en todas sus posibles sub-acciones (apoyarse en una pierna, elevar la otra para coger impulso, etc.), o integrarlo como componente de una actividad mayor, como puede ser un partido de futbol, indica que prototypical transitivity presupposes a conceptualization of events at a certain level of organization, rather than at subordinate or superordinate levels, (Taylor 1989:249). ${ }^{16}$ Esta tesis de Slobin viene confirmada, además de por la percepción psicológica que de ella tenemos, por diferentes hechos de la evolución lingüística seguida en el aprendizaje natural de una lengua y, en cualquier caso, hay una cierta unanimidad entre los lingüistas actuales en mantener que la Transitividad ocupa un lugar prototípico en la lengua humana, estructura por extensión otros procesos y tiene consecuencias semánticas y pragmáticas que resuelven la aparente separación entre "semántica”, "sintaxis" y "pragmática”, respondiendo a una gestalt-psycholinguistic notion of saliency, (Givón 1984:136). 17

15. En líneas generales, las diferentes propuestas metodológicas se han ido acompasando, en una relación simbiótica, a los sucesivos paradigmas lingüísticos; así se evidencia en los métodos audiovisuales inspirados en el estructuralismo de Bloomfield, en los estudios hechos en el modelo de Gramática Universal de Chomsky, en los modelos funcionales de lengua propuestos por Givón en 1979 y Halliday, y en la tipología de lenguas defendida por Comrie en 1984.

16. En este mismo sentido hay que incluir el ejemplo propuesto por Langacker ("Floyd broke the glass”), para evidenciar la no-correspondencia lógica entre los hechos y su representación lingüística, y cómo ésta supone la elaboración de un modelo cognitivo: Suppose Floyd's little sister, Andrea, has been teasing him mercilessly all morning. Angry and desirous of revenge, Floy picks up a hammer, swings it, and shatters Andrea's favourite drinking glass. The shards fly in all directions; one of them hits Andrea on the arm and cuts it, drawing blood. Hearing the commotion, their mother comes in and asks what happened. In response, Andrea utters the following inmortal words: FLOYD BROKE THE GLASS (Langacker, 1990:217).

17. Está claro que en ambos casos nos estamos refiriendo a la adquisición de una L1, pero anque más tarde matizaremos, de momento asumimos la posisición de McCarthy y Carter (1994:XI) a este respecto: 
El MLF defiende la naturaleza diagramática e icónica de la categoría verbo y postula una interconexión entre los diferentes ejes lingüísticos (principio de iconicidad); aunque ciertamente opta por la primacía del paradigmático, en cuanto que, al menos metodológicamente, es desde el análisis bottom-up de las estructuras definicionales de las entradas léxicas (semántica), desde donde se parte para establecer los modelos sintácticos de complementación; pero, finalmente, es la intersección de ambos ejes la que genera el esquema cognitivo conceptual como exponente de la significación gramatical entendida como una globalidad. Y en este sentido, el marco predicativo constituye un esquema cognitivo integrador de los diversos niveles lingüísticos. ${ }^{18}$

La relevancia pedágogica de esta interrelación de niveles lingüísticos exige cambiar la orientación seguida en la enseñanza del vocabulario, es necesario subrayar the text-creating and ideological foundation of lexical choice; to make vocabulary study more sensitive to a top-down view of language; to assist the job of relating higher order categories in the syllabus (such a text-type) to the micro-syllabus elements of grammar and lexis, (McCarthy y Carter, 1994:174).

Desde la consideración de la lengua como discurso y nuestra certeza de que una de las paradojas actuales en la lingüística aplicada a la enseñanza es que pese a la insistencia en el enfoque comunicativo sabemos más acerca de la lengua en los niveles léxicos y gramaticales que en el discursivo, ${ }^{19}$ nuestra propuesta didáctica, una vez seleccionado y organizado el corpus con los criterios de transitividad prototípica expuestos, se sustenta sobre dos pivotes: (i) el modelo cognitivo defendido por la semántica cognitiva; ${ }^{20}$ y (ii) la fórmula holística de presentación gráfica que hemos denominado Mapa Cognitivo Semántico (MCS).

we challenge that there are discontinuities between first and second language learning.. We prefer to underline the continuities and connections and argue that our discourse-based approach to language learning and teaching applies to both L1 and L2.

18. Estas posiciones teóricas del Modelo no son meras intuiciones sino evidencias sustentandas en datos empíricos y, en definitiva, responden a la concepción funcional de lengua: if a functional description of language means to describe Communicative Competence, this competence is incomplete without a description of the level of discourse, and if discourse-level constraints operate simultaneously with lexicogrammatical ones, is necessary to admit a discourse competence. (...) Grammar and vocabulary knowledge should involve how these aspects of linguistic form create discourse; in other words, linguistic competence cannot be separated from discourse competence, (McCarthy y Carter, 1994:174).

19. Consequently, many courses in language study are "bottom-up". They begin from the smallest, most manageable, most intensively studied units of language and work up to larger units. Sometimes the larger units are simply not reached, (...) wherever feasible, therefore, anlysis and description will be topdown or simultaneously bottom-up and top-down, (Carter, 1994:99).

20. The Cognitive Model Approach (Lakoff, 1987) es un modelo integrador que basándose en la Teoría Prototípica explicita el significado semántico en el nivel superficial y lo interrelaciona con el sintáctico y el pragmático. Además, conecta con la vivencia experiencial de cómo realmente se funciona en el proceso de adquisición de una lengua. Desde este paradigma cognitivo, se ha definido el aprendizaje lingüístico como un aspecto parcial del aprendizaje global que, en contra de otras concepciones (principalmente 
Limitándonos a la adquisición del fenómeno lingüístico que nos ocupa, Langacker (1992) propone dos modelos cognitivos básicos para la categorización de la transitividad prototípica:

i.. "The billiard-ball model": concebimos el mundo como si estuviera habitado por objetos individualizados, que ocupan un espacio en él. Algunos de estos objetos provocan movimiento al chocar con otros. La transmisión de esta energía produce el movimiento del objeto afectado.

ii. "The stage model”: otra concepción arquetípica es nuestra posición como observadores de los hechos que ocurren a nuestro alrededor, como si tuvieran lugar en un escenario y nosotros fuésemos expectadores. Concentramos nuestra atención en determinados aspectos del "continuum" que constituye la realidad, tendemos a organizar las escenas que contemplamos distinguiendo entre distintos "participantes" que interactúan dentro de un relativamente estable "escenario".

Los "eventos" representarían como "trozos" de estas acciones que se sitúan en un mismo eje temporal. Así pues, estos modelos idealize an essential aspect of our ongoing experience: the observation of sequences of external events, each involving the interactions of participants within a setting (Langacker, 1990:215).

La combinación de estos modelos -"bola de billar", "teatral” y los roles semánticos de los participantes- da lugar a una concepción compleja que podría reflejarse del siguiente modo y que representaría the normal observation of a prototypical action (Langacker, 1990:216):

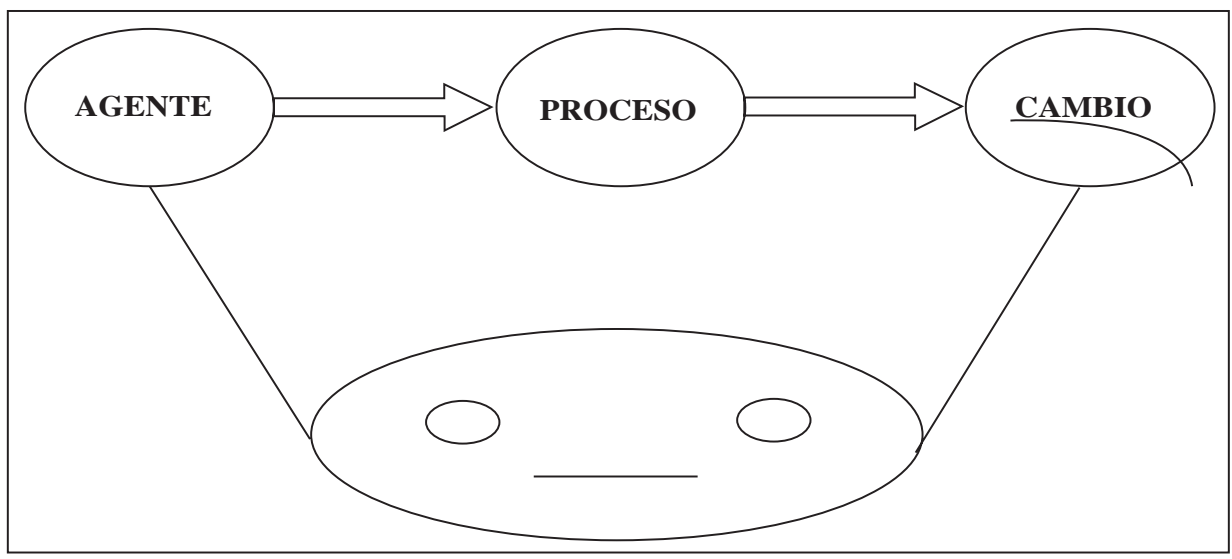

Esquema del MCI para la adquisición de la transitividad prototípica

las defendidas por los modelos conductistas y las teorías mentalistas), fundamenta su teoría del conocimiento en lo que se denomina "realismo experiencial": el prototipo representa el "nivel básico" desde el cual, y, a partir de la experiencia de nuestra interrelación con el entorno como entes corpóreos, se forman y desarrollan los esquemas cognitivos de diversos niveles que hacen posible el conocimiento del mundo de los objetos mediante su categorización. 
Este modelo cognitivo refleja la estructura típica de una oración simple, que conceptualiza una acción. Este proceso prototípico representa la interacción de un agente con un paciente, el primero se selecciona como sujeto de la oración, y el segundo como su objeto directo. Pese a la aparente simplicidad, el proceso de codificación lingüística es bastante selectivo: el evento comprende una intrincada red de interacciones que incluye numerosas entidades con posibilidad de aparecer en la frase como participantes; no obstante, sólo algunas de estas interacciones y participantes son hechas explícitas en las frases: (...) clearly, then, the objective properties of a situation do not mechanically determine the grammatical, (Langacker, 1990:217)

Siguiendo estas formulaciones, si admitimos el valor prototípico del lexema verbal transitivo y, como corolario, su importancia en la estructuración de los distintos niveles de lengua, su esquema conceptual cognitivo debe resultar clave en el proceso de adquisición de una L2 puesto que, como ha demostrado la teoría de los esquemas, se da la transferencia tanto de los esquema de contenidos (en este caso, ámbito experiencial básico), como de los esquemas formales o estructura retórica del discurso ( en este caso foregrounding meaning of discourse). ${ }^{21}$ Precísamente, uno de los grandes logros del MLF ha sido el de aportar una concepción de esquema-cognitivo-conceptual aplicable al entorno linguiístico, demostrando empíricamente que están "linguistically motivated" y son el resultado de la confluencia de los ejes lingüísticos, tras el análisis "bottom-up" de las definiciones léxicas, de acuerdo con el método de "stepwise lexical decomposition" propuesto por Dik, (Cf. Faber y Mairal, 1995). En efecto, a partir del estatus prototípico concedido al archilexema, se genera una amplia tipología de esquemas que reflejan la propia estructuración jerárquica del léxico organizada en diversos niveles (macro-campos, campos, dimensiones y lexemas).

Desde esta perspectiva, nuestra propuesta de aislar un corpus funcional de lexemas verbales prototípicamente transitivos, se complementa con el recurso didáctico que hemos denominado MCS. ${ }^{22}$ Se trata de reorganizar en dimensiones el corpus ya seleccionado con los criterios de prototipicidad ya expuestos. La arquitectura resultante se refleja de modo gráfico en una tipología de estos mapas que representarían esquemas conceptuales de niveles dimensionales en los que los argumentos vendrían representados por los archilexemas respectivos, que ocuparían el vértice jerárquico e

21. Esto que se puede afirmar de una L1 es aplicable con más razón a la adquisición de una L2. En efecto, de acuerdo con la teoría de los esquemas existe entre ambas un proceso de transferencia o interrelación entre las habilidades lingüísticas conceptuales y las estrategias procedimentales: In the top-down view of second language acquisition (...) Everything in the (learner) prior linguistic knowledge ("linguistic schemata") and level of proficiency in the second language is importatnt but the learner's prior background knowlege of the content area of the (discourse) ("content schemata") as well as the rhetorical structure of the text ("formal schemata") are also important (Coady (1979). Citado por Carrell, 1983).

22. Para un estudio más pormenorizado del MCS véase L. Rodríguez, 1997b El Mapa Cognitivo Semántico: una metodología integrada para la enseñanza/aprendizaje del inglés como L2 basada en el análisis del léxico mental propuesto por el Modelo Lexemático Funcional. Córdoba: UNED. 
introducirían el universo sintáctico/semántico/pragmático, y los hipónimos, como lexemas nucleares que integrarían la dimensión que cada mapa representaría. La relación entre las entidades argumentales es de carácter jerárquico, heredando los hipónimos las características argumentales de su hiperónimo inmediato, y, todos, las del archilexema que establece la dimensión.

Esta estructuración jerarquizada, aunque procura un aprendizaje en el que se implementan las estrategias de carácter deductivo ("top-down") en cuanto facilita las inferencias basadas en una serie de principios gramaticales previos expresados por el archilexema que domina la dimensión, pretende, sin embargo, seguir una doble ruta cognitiva en la que también se incluye el aprendizaje inductivo derivado del estudio individualizado de lexemas, al objeto de facilitar la asimilación de reglas (botton-up) y las conexiones cognitivas con otros campos del lexicón.

\section{Conclusiones}

La importancia del componente léxico en el análisis lingüístico puesto de manifiesto por la últimas corrientes lingüísticas, ha traido consigo aplicaciones didácticas para la eneseñanza aprendizaje de una L2. Existe una necesidad de considerar en detalle las bases de nuestra presentación del vocabulario. A tal efecto, no puede seguir presentándose como simple ejemplificación de las estructuras sintácticas ni seguir ocupando una función menor en el nivel léxico. Si la lengua es comunicación y ésta se establece mediante la competencia discursiva y queremos recuperar la centralidad del léxico en el proceso de enseñanza/aprendizaje, éste debe proyectarnos e integrarse en otros macro-niveles. Desde la perspectiva del MLF, proponemos aislar un corpus nuclear de verbos prototípicamente transitivos porque lingüística y psicológicamente responden a esta demanda de globalización y representa un amplio ámbito experiencial. Siguiendo el proceso categorial defendido por la teoría cognitiva, proponemos a tal efecto la estructuración de este corpus en una serie de MCSs de niveles dimensionales, introducidos por los esquemas cognitivos conceptuales derivados del análisis lingüístico de los archilexemas, reflejados en los marcos predicativos y en conexión con una base da datos multifuncional donde se recoja toda la información lingüística al respecto.

En efecto, una tipología básica de estos esquemas cognitivos conceptuales puede ser un poderoso instrumento de economía cognitiva en el aprendizaje de una lengua. En especial el de una segunda lengua, puesto que una teoría cognitiva de adquisición de la misma, implica el aprovechar la ventaja que supone la base conceptual/lingüística que, de acuerdo con la Interdependence Hypothesis defendida por Cummins (1984) y Bialystock y Ryan (1985) facilita los procesos de transferencia. 
LUIS RODRÍGUEZ GARCÍA

\section{Bibliografía}

AITCHISON, J. 1987. Words in the Mind: An Introduction to the Mental Lexicon. Oxford: Basil Blackwell.

ALLERTON, D. J. 1982. Valency and the English Verb. London: Academic Press.

BIALYSTOK, E. y RYAN, E.B. (1985). "A metacognitive framework for the development of first and second language skills" en D.L. Forrest-Pressley,G. E. Mackinnon, and T.G. Waller, (Eds.), Metacognition, cognition, and human performance (pp.207-245). Orlando, Florida: Academic

CANO, AGUILAR, R.. 1981. Estructuras sintácticas transitivas en el español actual Madrid: Gredos.

CARTER, R. 1993.'Describing knowledge about language: pupils, teachers and the LINC programme", en Techniques of Description. Sinclair, J.M., M. Hoey y G. Fox, Eds.. London and New York: Routledge.

CARREL, P.L. y J. C. EISTERHOLD. 1993. "Schema Theory ans ESL reading pedagogy", en Carrel P. L., J. Devine and D. Eskey Eds. Interactive Approaches to Second Language Reading. Cambridge University Press.

COSERIU, E. 1977. Principios de semántica estructural. Madrid: Gredos.

CROFT. H. 1990. "Possible verbs and the strucure of events" en S. Tsohatzidis (ed.), Meanings and prototypes: studies in linguistic categorization. 48-73.

DIK, S.C. 1980. Studies in Funtional Grammar. London: Academic Press.

DIK, S.C. 1983. Advances in Functional Grammar. Dordrecht: Foris Publications.

DIK, S.C. 1978a. Functional Grammar. Dordrecht: Foris Publications.

DIK, S.C. 1986. 'Linguistically motivated knowledge representation'. Working Papers in Functional Grammar no. 9. Amsterdam: University of Amsterdam.

DIK, S.C. 1989. The Theory of Functional Grammar. Part I: The Structure of the Clause. Dordrecht: Foris Publications.

FABER, P. y MAIRAL, R. 1994a. 'Methodological underpinnings for the construction of a functional lexicological model'. Miscelánea. A Journal of English and American Studies 15: 193-217.

FABER, P. y MAIRAL, R. en prensa.'Semantic Frames and Dimenssions: Towards a Unified Approach".

FELBAUM, C. 1991. "English Verbs as a Semantic Net". International Journal of Lexicography 3-4: 279-301.

GIVON, T. 1984. Syntax. A Functional Typological Introduction. Volume I. Amsterdam/Philadelphia: John Benjamins.

GOOSSENS, L. 1990b. 'Transitivity and the Treatment of (Non)-Prototypicality in FG'. Paper presented at the Fourth International Conference in FG, June 1990, Copenhagen.

HALLIDAY, M.A.K. 1985. Explorations in the functions of language. London: Edward Arnold. 
HOPPER, P. J. y THOMPSON, S. A. 1980. "Transitivity in grammar and discourse". Language, 56.3.

JOHNSON, M. 1987. The Body in the Mind. Chicago: University of Chicago Press.

LANGACKER, R. W. 1987. Foundations of Cognitive Grammar. Stanford: Stanford University Press.

LANGACKER, R. W. 1990. "Settings, participants, and grammatical relations" en S.Tsohatzidis (ed.), Meanings and prototypes: studies in linguistic categorization, (pp. 213-239).

MACKAY, S. 1980. "Teaching the syntactic, semantic and pragmatic dimensions of verbs". TESOL Quaterly, 14.

MAIRAL, R. en prensa. 'Parámetros para la organización de una Sintaxis Léxica Funcional' en J. Martín Arista (ed.), Estudios de Gramática Funcional. Zaragoza: Servicio de Publicaciones de la Universidad de Zaragoza.

MARTIN MINGORANCE, L. 1990. `Functional Grammar and Lexematics’ in J. Tomaszczyk and B. Lewandowska-Tomaszczyk (eds.), Meaning and Lexicography. Amsterdam/ Philadelphia: John Benjamins.

MARTIN MINGORANCE, L. 1995. "Lexical logic and structural semantics. Methodological underpinnings in the structuring of a lexical database for natural language processing."

MCCARTHY, M. y RONALD CARTER. 1994. Language as discourse. Perspectives for Language teaching. New York: Longman.

PINKER, S. 1994." How could child use verb syntax to learn verb semantics?". Lingua92:377-410. North Holland.

SINCLAIR, J. M. 1993. "Written Discourse Structure", en Techniques of Description. Sinclair, J.M., M. Hoey y G. Fox, Eds. London and New York: Routledge.

SLOBIN, D.I. 1980. "Cognitive Prerequisites for the Development of child Language", en Ferguson Slobin (eds). Studies of Child Language Development. New York. Holt

STUBBS, M. 1986a. "Language Development, lexical competence and nuclear Vocabulary" en Educational Linguistics. Oxford: Blackwell. 98-115.

TAYLOR, J.R. 1989. Linguistic Categorization: Prototypes in Linguistic Theory. Oxford: Clarendon Press.

WOTJAK, B. 1993. "El léxico-encrucijada a la lingüística teórica y aplicada, de metodologías y disciplinas distintas". Ponencia desarrollada en el Congreso de Granada, enero de 1993. 